\title{
The relationship between water intake and foetal growth and preterm delivery in a prospective cohort study
}

\author{
J Michael Wright ${ }^{1 *}$, Caroline S Hoffman², David A Savitz ${ }^{3}$
}

\begin{abstract}
Background: Interpretation of previous associations between water intake and adverse birth outcomes is challenging given that amount and type of water consumed can be non-specific markers of exposure or underlying behavioural characteristics. We examined the relationship between water intake measures and adverse birth outcomes in participants from three study sites in the United States.

Methods: Using a prospective cohort study, we examined daily intake of bottled, cold tap, total tap, and total water in relation to birth weight and risk of small-for-gestational-age (SGA) among term births and risk of preterm delivery.

Results: Based on water consumption data collected between 20-24 weeks of gestation, the adjusted mean birth weight was 27 (95\% confidence interval [Cl]: -34, 87), 39 (95\% Cl: -22, 99), and 50 (95\% Cl: -11, 110) grams higher for the upper three total water intake quartiles $(>51-78,>78-114$, and $>114$ ounces/day) compared to the lowest quartile ( $\leq 51$ ounces/day). Adjusted birth weight results were similar for bottled water, cold tap water, and total tap water intake. An exposure-response gradient was not detected for either preterm delivery or SGA with increasing total water intake and total tap water intake, but adjusted relative risks for all three upper quartiles were below 1.0 (range: 0.6-0.9) for SGA.
\end{abstract}

Conclusion: These data suggest that high water intake may be associated with higher mean birth weight following adjustment for confounding.

\section{Background}

Water consumption is critical for metabolism, temperature regulation, transporting nutrients and wastes, and tissue maintenance. Water intake is also important for pregnant women with oligohydramnios and those at risk of developing uteroplacental insufficiency [1]. Few epidemiological studies have addressed the role of water intake on adverse reproductive outcomes with most of these focusing on the effect of specific contaminants such as disinfection by-products. Savitz et al [2] reported an inverse association between increased water intake and risk of preterm delivery (PTD) (ie, < 37 gestational weeks) and low birth weight infants. Compared to those reporting no daily water intake, odds

\footnotetext{
* Correspondence: wright.michael@epa.gov

'National Center for Environmental Assessment, US Environmental Protection Agency, Cincinnati, Ohio, USA

Full list of author information is available at the end of the article
}

ratios (ORs) were 0.5 and 0.6 for $>4$ glasses/day for small for gestational age (SGA) and PTD, respectively. Relative to low intake (1-7 glasses/week), Aggazzotti et al [3] showed little evidence of an association between high intake of tap water (> 35 glasses/week) and risk of SGA or PTD (ORs = 1.0 and 1.1, respectively). Other studies have shown a decreased risk of spontaneous abortion [4] and cardiac anomalies [5] with increased bottled water intake. Given that water intake is a non-specific marker of exposure, it is not clear if these results are due to residual confounding or actual effects of water ingestion.

Using a prospective cohort study, we examined birth weight and risk of SGA among term births and risk of PTD in relation to daily bottled, cold tap, total tap and total water intake. The primary study hypothesis examined whether water intake is associated with measures of foetal growth and protective of adverse birth outcomes. 


\section{Methods}

\section{Study design and population}

The study population included 2766 pregnant women enrolled in a prospective cohort study conducted from December 2000-May 2004 across three study sites in the United States. Participants were enrolled early in pregnancy ( $\leq 12$ weeks' gestation) or while planning to become pregnant. Eligible subjects included those who were $\geq 18$ years of age, who did not have any fertility treatment for the study pregnancy, and who intended to deliver in the study area. Additional details on study design and recruitment have been published elsewhere $[6,7]$. The Institutional Review Boards at the University of North Carolina, University of Tennessee and the University of Texas approved the study protocols; participants gave informed consent.

The sample size for the PTD analysis was 2039 pregnancies after the following exclusions: 259 with a missing or incomplete baseline interview, 347 with a pregnancy loss, 90 that were lost to follow-up, 16 with repeat live births, eight with multiple births, and seven with missing information on date of birth or birth weight. 1854 live births were available for the birth weight analysis, and 1783 live term births were available for the SGA analysis due to missing information on maternal race/ethnicity, births with a reported maternal race of "Indian," "Asian/Pacific Islander," or "Other," and births delivered at $<25$ or $>42$ weeks' gestation.

\section{Assessment of foetal growth and PTD}

Infant date of birth, birth weight, and gender were obtained from medical records for $43 \%$ of live births, from vital records for $57 \%$, and from participant selfreport for $<1 \%$. Self-reported last menstrual period (LMP) and an early ultrasound (scheduled between gestational weeks 6-7 and no later than 14 weeks), both obtained during the first trimester, were combined with infant date of birth to estimate gestational age at birth. Gestational age derived from LMP was used for the majority of subjects (81\%) unless the LMP date was incomplete (1\%) or differed by more than \pm 7 days from the ultrasound-based estimate of gestational age (18\%), in which case the ultrasound estimate was used. SGA was defined as an infant with a birth weight below the tenth percentile for gestational age at birth, gender, maternal race/ethnicity (non-Hispanic white, non-Hispanic black, or Hispanic), and parity based on United States population estimates $[8,9]$.

\section{Assessment of exposures and confounding factors}

Data on exposures and potential confounding factors were collected via telephone interviews before 16 weeks of gestation (baseline interview) and between 20-24 weeks of gestation (follow-up interview). The interviews included detailed questions about the pregnancy, maternal health, demographic information, behavioural characteristics, and water use practices. Maternal health characteristics included pre-pregnancy body mass index (BMI) defined as weight $/$ height ${ }^{2}$ and categorized according to the Institute of Medicine's [10] guidelines: low $\left(<19.8 \mathrm{~kg} / \mathrm{m}^{2}\right)$, normal $\left(19.8-26.0 \mathrm{~kg} / \mathrm{m}^{2}\right)$, overweight $\left(26.1-29.0 \mathrm{~kg} / \mathrm{m}^{2}\right)$, and obese $\left(>29.0 \mathrm{~kg} / \mathrm{m}^{2}\right)$. Behavioural variables included recreational exercise, smoking, intake of caffeine, vitamins, alcohol, and illicit drugs. Caffeine intake from beverages (ie, coffee, tea, and soda) was estimated and then categorized using the cut points $150 \mathrm{mg} /$ day and $300 \mathrm{mg} /$ day [11].

At each interview, study participants were asked how many bottles of water and glasses/cups of cold tap water, hot tap water and tap water-based drinks (including juice, coffee, tea, and other beverages they made from tap water) they consumed each day during a typical week. Participants were asked to define their glass or cup sizes according to three options: small (0.1-0.3 L), medium (0.4-0.6 L), or large (0.7-1.0 L) for cold tap and bottled water and small (0.1-0.3 L), medium (0.3-0.5 L), or large (0.5-0.7 L) for hot water. The midpoint for each size range was used to estimate water consumption in ounces/day. Bottled water included spring water, mineral water, distilled water, sparkling water or any water purchased in bottles or plastic jugs or obtained from a water cooler. Bottled water intake was calculated as the average amount based on reported container sizes: small (8-12 ounces), medium (14-24 ounces), and large (26-34 ounces). Among the women working outside the study area (8\%), the tap water ingestion question was asked separately for consumption at home and at work. This resulted in a higher average total amount for this group. We, therefore, deflated their cold tap water consumption totals by $15.3 \%$ and hot tap water consumption by $18.2 \%$ for those reporting work and home totals separately to make their mean values equal to those women who reported the aggregated amount.

Follow-up data and an average of the baseline and follow-up data were used to examine the following exposure measures: cold tap water intake, total tap water (cold and hot) intake, bottled water intake, and total water (tap and bottled) intake. Water use measures were divided into quartiles and analyzed using the lowest quartile as the referent. Based on self-reported data collected during the follow-up questionnaire, women were classified into the following quartiles for total water intake: 0-51 (referent), > 51-78, > 78-114, and > 114 ounces/day. Women were classified into the following quartiles for total tap water intake: 0-30 (referent), > 30$61,>61-96$, and $>96$ ounces/day. Due to limited data on bottled water intake in the population, this variable 
was dichotomized to allow comparison of any versus no bottled water intake.

\section{Statistical analysis}

We calculated risk ratios (RRs) and 95\% confidence intervals (CIs) for SGA and preterm delivery based on various water consumption measures using Poisson regression with robust error variance. The association with term birth weight was examined using linear regression. We considered potential confounding variables that were associated with the outcomes and were independently associated with water use exposures, but were not intermediates in the causal pathway between exposure and disease: maternal age, race/ethnicity, education level, annual household income, employment status, marital status, pre-pregnancy BMI, parity, alcohol consumption, smoking status, caffeine intake, vitamin intake, recreational activity, swimming, infant gender, season of birth, and study site. Maternal education, race/ethnicity, income, infant gender, parity, pre-pregnancy BMI, smoking, vitamin intake, employment during pregnancy, and study site were retained in multivariate models as confounders using a change-inestimate (10\% change) backwards elimination approach. We examined the extent that confounding impacted the birth weight results among those variables retained in the backwards elimination model. Relative to the unadjusted models for total water intake from the follow-up data, we examined percent change-in-estimate for the linear regression models for the confounders that were retained in multivariate models.

\section{Results}

The mean birth weight in the total population $(\mathrm{n}=$ 2039) was 3382 grams, with $9 \%(n=192)$ born preterm (Table 1). Among the 1854 term births, $5 \%(n=85)$ were classified as SGA. Lower mean birth weights were found among infants born to mothers who were nonHispanic black, of younger age, were not married, had lower BMI, were less educated, and had an annual household income $<\$ 30,000$. Large differences in mean birth weight were also detected between Site 1 versus Site 3 (165 grams), non-smokers versus smokers (325 grams), and vitamin users versus non-users (159 grams). Several factors were associated with both higher mean birth weight and higher water intake. For example, mothers of non-Hispanic white ethnicity, higher BMI, and those who consumed vitamins tended to have larger infants and drink larger amounts of total water, so that adjustment for these factors attenuated the association between water intake and birth weight. Using a changein estimate analysis, we examined the individual contribution of various confounders in the birth weight model for total water intake based on the follow-up data.
Table 1 Characteristics of the study population recruited from three US cities during 2000-4

\begin{tabular}{|c|c|c|c|c|c|c|}
\hline \multirow[t]{2}{*}{ Population characteristics } & \multirow[b]{2}{*}{$\mathbf{n}$} & \multirow[b]{2}{*}{$\%$} & \multirow{2}{*}{$\begin{array}{l}\text { PTD } \\
\%\end{array}$} & \multirow{2}{*}{$\begin{array}{l}S^{S G A}{ }^{a} \\
\%\end{array}$} & \multicolumn{2}{|c|}{ Birth weight (g } \\
\hline & & & & & Mean & SD \\
\hline Total population & 2039 & 100 & 9 & 5 & 3382 & 586 \\
\hline \multicolumn{7}{|l|}{ Maternal race/ethnicity } \\
\hline Non-Hispanic white & 1169 & 57 & 7 & 5 & 3486 & 596 \\
\hline Non-Hispanic black & 609 & 30 & 12 & 7 & 3167 & 545 \\
\hline Hispanic & 185 & 9 & 9 & 6 & 3427 & 632 \\
\hline Other & 73 & 4 & 7 & - & 3400 & 459 \\
\hline Missing & 3 & & & & & \\
\hline \multicolumn{7}{|l|}{ Maternal age (years) } \\
\hline$<25$ & 599 & 29 & 11 & 7 & 3255 & 558 \\
\hline $25-29$ & 657 & 32 & 8 & 6 & 3393 & 572 \\
\hline $30-34$ & 564 & 28 & 7 & 4 & 3486 & 587 \\
\hline$\geq 35$ & 219 & 11 & 11 & 6 & 3425 & 642 \\
\hline
\end{tabular}

Highest maternal education level

$\begin{array}{lllllll}\text { High school or less } & 573 & 28 & 13 & 9 & 3235 & 616\end{array}$

$\begin{array}{lllllll}\text { Some college } & 440 & 22 & 10 & 5 & 3340 & 600\end{array}$

$\begin{array}{lllllll}\text { College degree or } & 1025 & 50 & 7 & 4 & 3482 & 544\end{array}$

higher

Maternal smoking

$\begin{array}{lllllll}\text { Yes } & 99 & 5 & 16 & 12 & 3073 & 578\end{array}$

$\begin{array}{llllll}\text { No } & 1940 & 95 & 9 & 5 & 3398\end{array}$

Maternal alcohol use

$\begin{array}{lllllll}\text { Yes } & 32 & 2 & 9 & 6 & 3410 & 702\end{array}$

$\begin{array}{lllllll}\text { No } & 2007 & 98 & 6 & 6 & 3381 & 585\end{array}$

Pre-pregnancy BMI $\left(\mathrm{kg} / \mathrm{m}^{2}\right)$

$\begin{array}{lllllll}<19.8 & 232 & 11 & 8 & 9 & 3291 & 535\end{array}$

$\begin{array}{lllllll}19.8-25.9 & 1016 & 50 & 8 & 5 & 3410 & 553\end{array}$

$\begin{array}{lllllll}26.0-29.9 & 333 & 16 & 7 & 6 & 3421 & 535\end{array}$

$\begin{array}{lllllll}>29.9 & 407 & 20 & 14 & 5 & 3349 & 713\end{array}$

$\begin{array}{lllllll}\text { Missing } & 51 & 3 & 10 & 16 & 3255 & 771\end{array}$

Vitamin use

$\begin{array}{lllllll}\text { Yes } & 1027 & 50 & 7 & 5 & 3461 & 573\end{array}$

$\begin{array}{lllllll}\text { No } & 1012 & 50 & 11 & 7 & 3302 & 590\end{array}$

Caffeine intake (mg/day)

$\begin{array}{lllllll}\text { None } & 519 & 25 & 9 & 4 & 3410 & 613\end{array}$

$\begin{array}{lllllll}1-150 & 468 & 23 & 8 & 5 & 3385 & 557\end{array}$

$\begin{array}{lllllll}151-300 & 387 & 19 & 11 & 6 & 3344 & 617\end{array}$

$\begin{array}{lllllll}>300 & 665 & 33 & 9 & 7 & 3380 & 567\end{array}$

Marital status

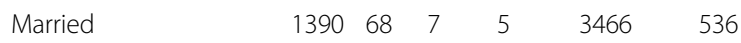

$\begin{array}{lllllll}\text { Not married } & 648 & 32 & 13 & 8 & 3200 & 646\end{array}$

Missing

Parity

$\begin{array}{lllllll}\text { Nulliparous } & 991 & 49 & 10 & 6 & 3323 & 591\end{array}$

$\begin{array}{lllllll}\text { Parous } & 1048 & 51 & 8 & 4 & 3438 & 577\end{array}$

Employed during past 4 months

$\begin{array}{lllllll}\text { Yes } & 1430 & 70 & 9 & 5 & 3372 & 580\end{array}$

$\begin{array}{lllllll}\text { No } & 608 & 30 & 9 & 7 & 3406 & 602\end{array}$

Annual household income (\$)

$\begin{array}{lllllll}<30,000 & 637 & 31 & 12 & 7 & 3245 & 629\end{array}$

$\begin{array}{lllllll}30,001-60,000 & 535 & 26 & 7 & 7 & 3437 & 545\end{array}$ 
Table 1 Characteristics of the study population recruited from three US cities during 2000-4 (Continued)

\begin{tabular}{lllllll}
\hline 60,001-80,000 & 321 & 16 & 6 & 5 & 3460 & 498 \\
$>80,000$ & 465 & 23 & 8 & 3 & 3504 & 571 \\
$\quad$ Missing & 81 & 4 & 20 & 10 & 3078 & 614 \\
$\begin{array}{l}\text { Recreational activity } \\
\quad \text { Yes }\end{array}$ & 1109 & 54 & 9 & 6 & 3423 & 572 \\
$\quad$ No & 930 & 46 & 10 & 5 & 3332 & 600 \\
$\begin{array}{l}\text { Vigorous recreational activityc } \\
\text { Yes }\end{array}$ & & & & & \\
$\quad$ No & 401 & 20 & 8 & 7 & 3412 & 559 \\
Swimming & 1638 & 80 & 9 & 5 & 3374 & 593 \\
$\quad$ Yes & & & & & & \\
$\quad$ No & 651 & 58 & 6 & 6 & 3471 & 549 \\
Infant gender & 1388 & 32 & 10 & 6 & 3340 & 599 \\
$\quad$ Male & & & & & & \\
Female & 1045 & 51 & 9 & 7 & 3419 & 585 \\
Study site & 994 & 49 & 9 & 5 & 3343 & 586 \\
Site 1 & & & & & & \\
Site 2 & 929 & 46 & 6 & 5 & 3459 & 581 \\
Site 3 & 761 & 37 & 11 & 6 & 3329 & 593 \\
\hline
\end{tabular}

${ }^{\mathrm{a}}$ Term births only

${ }^{\mathrm{b}}$ Any recreational physical activity or exercise, such as brisk walking, jogging, swimming, biking, tennis, soccer, or dancing

'Any recreational physical activity or exercise, such as brisk walking, jogging, swimming, biking, tennis, soccer, or dancing that caused large increases in breathing and heart rate

$\mathrm{SGA}=$ small for gestational age; $\mathrm{PTD}=$ preterm delivery $\mathrm{SD}=$ standard deviation; $\mathrm{BMI}=$ body mass index

Compared to the univariate results, the largest average change-in estimates across the highest three exposure quartiles were swimming (39\%), maternal race/ethnicity (31\%), annual household income (21\%), education (20\%), marital status (20\%), and vitamin intake (17\%).

\section{Birth weight}

Compared to the lowest quartile, the unadjusted mean birth weight in grams was higher for the upper three quartiles of total water intake, 60 (95\% CI: -1, 122), 67 (95\% CI: 5, 128), and 83 (95\% CI: 21, 145) grams, respectively (Table 2). Following adjustment for confounding (by study site, household income, maternal education, maternal race/ethnicity, infant gender, parity, pre-pregnancy BMI, smoking, vitamin use, and employment during pregnancy), the respective adjusted differences in mean birth weight in grams were reduced to 27 (95\% CI: -34, 87), 39 (95\% CI: -22, 99), and 50 (95\% CI: -11, 111) grams compared to the lowest quartile. When total water intake was examined as a continuous measure (per 20 ounce/day increased intake), the adjusted increase in mean birth weight was 7.3 (95\% CI: -0.8, 15.5) grams. Adjusted results were similar in magnitude for the upper quartiles of cold tap water intake, total tap water intake, and total water intake for both the follow-up data and an average of the follow-up and baseline data. Compared to no bottled water intake, the adjusted mean birth weight for bottled water consumers was 31 grams (95\% CI: -20 , 82) based on follow-up data and 43 grams (95\% CI: -27 , 113) based on an average of the follow-up and baseline data.

\section{SGA}

RRs and 95\% CIs comparing women who reported drinking $>51-78,>78-114$, and $>114$ versus $0-51$ ounces of total water per day at follow-up were $0.7(0.4$, $1.2), 0.6(0.3,1.0)$, and $0.8(0.5,1.4)$, indicative of a decreased risk of SGA with increased water consumption above the first quartile but no gradient thereafter (Table 3). Results were similar following adjustment for confounding: $0.8(0.4,1.4), 0.6(0.3,1.0)$, and $0.9(0.5$, 1.6), respectively. Relative to the lowest quartile, adjusted RRs were slightly higher for cold tap and total tap water intake especially for the average follow-up and baseline data. Relative to women not drinking bottled water, the adjusted RR for SGA was 0.9 (95\% CI: 0.5, 1.4) based on the follow-up data and $1.4(0.6,3.0)$ based on the average follow-up and baseline data.

\section{PTD}

As shown in Table 4, RRs and 95\% CIs for PTD comparing women who reported drinking $>51-78,>78$ 114 , and $>114$ versus $0-51$ ounces of total water per day based on the follow-up data were $1.0(0.7,1.6), 1.0$ $(0.7,1.6)$, and $1.2(0.8,1.8)$. RRs were slightly larger following adjustment for confounding: $1.2(0.7,1.9)$, $1.1(0.7,1.8)$, and $1.4(0.9,2.2)$, respectively. RRs and 95\% CIs for PTD for the cold tap and total tap water quartiles were generally below 1.0 compared to the lowest quartile for follow-up data and average followup and baseline data. Relative to women not drinking bottled water, the adjusted RR for any bottled water intake was $1.2(95 \%$ CI: $0.8,1.8)$ for the follow-up data and 0.8 (95\% CI: $0.5,1.4)$ based on the average baseline and follow-up data.

\section{Discussion}

Previous epidemiological studies of disinfection by-products in drinking water have shown a possible increased risk of impaired foetal growth with increasing exposure but a decreased risk of PTD [12-16]. Hoffman et al [13] postulated that the decreased risk of PTD may be partially due to a protective effect of higher water intake on pregnancy outcomes, but this has rarely been examined. Overall, the adjusted SGA results in our study were largely null although the RRs for follow-up data on total tap water and total water intake were consistently below 1.0 (range 0.6-0.9) for the higher water exposure categories. The adjusted PTD findings were also largely null 
Table 2 Birth weight results for daily bottled, cold tap, total tap, and total water intake

\begin{tabular}{|c|c|c|c|c|c|c|c|}
\hline \multirow[b]{3}{*}{ Exposure } & \multirow[b]{3}{*}{ n (\%) } & \multicolumn{4}{|c|}{ Follow-up data } & \multirow{2}{*}{\multicolumn{2}{|c|}{$\begin{array}{l}\text { Average of baseline } \\
\text { and follow-up data } \\
\text { Adjusted }^{\mathrm{a}}\end{array}$}} \\
\hline & & \multicolumn{2}{|c|}{ Unadjusted } & \multicolumn{2}{|c|}{ Adjusted $^{\mathrm{a}}$} & & \\
\hline & & $\bar{\beta}$ & $95 \% \mathrm{Cl}$ & $\beta$ & $95 \% \mathrm{Cl}$ & $\beta$ & $95 \% \mathrm{Cl}$ \\
\hline \multicolumn{8}{|l|}{ Bottled water } \\
\hline None & $448(25 \%)$ & Ref & & Ref & & Ref & \\
\hline Any & 1329 (75\%) & 12 & $(-38,63)$ & 31 & $(-20,82)$ & 43 & $(-27,113)$ \\
\hline \multicolumn{8}{|c|}{ Cold tap water (ounces) ${ }^{b}$} \\
\hline $0-27$ & $424(24 \%)$ & Ref & & Ref & & Ref & \\
\hline$>27-53$ & $455(25 \%)$ & 12 & $(-50,74)$ & 9 & $(-53,72)$ & 25 & $(-38,88)$ \\
\hline$>53-91$ & $444(25 \%)$ & 74 & $(11,136)$ & 52 & $(-11,116)$ & 44 & $(-19,107)$ \\
\hline$>91$ & $453(26 \%)$ & 77 & $(14,139)$ & 49 & $(-14,111)$ & 65 & $(2,128)$ \\
\hline Per 20 ounce & & 12.3 & $(3.9,20.7)$ & 8.5 & $(0.1,16.9)$ & 8.5 & $(-1.5,18.5)$ \\
\hline \multicolumn{8}{|c|}{ Total tap water (ounces) ${ }^{b}$} \\
\hline $0-30$ & $443(25 \%)$ & Ref & & Ref & & Ref & \\
\hline$>30-61$ & $445(25 \%)$ & 41 & $(-21,103)$ & 44 & $(-18,106)$ & 10 & $(-52,73)$ \\
\hline$>61-96$ & $496(28 \%)$ & 100 & $(40,161)$ & 78 & $(17,139)$ & 34 & $(-30,97)$ \\
\hline$>96$ & $392(22 \%)$ & 85 & $(21,149)$ & 43 & $(-21,107)$ & 46 & $(-17,109)$ \\
\hline Per 20 ounce & & 12.0 & $(4.0,20.1)$ & 6.8 & $(-1.3,15.0)$ & 5.3 & $(-4.3,15.1)$ \\
\hline \multicolumn{8}{|c|}{ Total water (ounces) ${ }^{b}$} \\
\hline $0-51$ & $452(25 \%)$ & Ref & & Ref & & Ref & \\
\hline$>51-78$ & $439(25 \%)$ & 60 & $(-1,122)$ & 27 & $(-34,87)$ & 10 & $(-50,71)$ \\
\hline$>78-114$ & $442(25 \%)$ & 67 & $(5,128)$ & 39 & $(-22,99)$ & 55 & $(-6,116)$ \\
\hline$>114$ & $441(25 \%)$ & 83 & $(21,145)$ & 50 & $(-11,111)$ & 37 & $(-25,98)$ \\
\hline Per 20 ounce & & 9.8 & $(1.6,18.1)$ & 7.3 & $(-0.8,15.5)$ & 5.2 & $(-4.7,15.2)$ \\
\hline
\end{tabular}

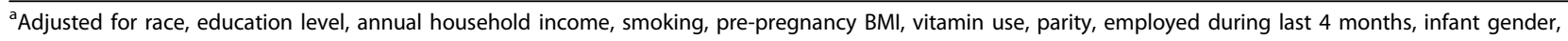
and study site

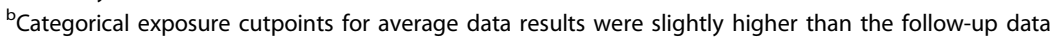
$\mathrm{BMI}=$ body mass index.

with most RRs below the null value of 1.0. For both the follow-up and average of follow-up and baseline data analyses, we observed a slight increased risk of PTD in the high total water intake group (RR's $=1.2$ and 1.4) relative to those in the low intake category.

To the best of our knowledge, this is the first study to examine the relationship between water intake and birth weight in a prospective epidemiological study. We saw some evidence of an exposure-response relationship with mean birth weight differences ranging from 27-50 grams with increasing total water intake compared to the lowest quartile after adjustment. We found results similar in magnitude for the cold tap water and total tap water intake measures based on the follow-up data. Similar results were found for all three exposure measures based on an average of baseline and follow-up questionnaire data including suggestion of an exposureresponse relationship for cold tap and total tap water intake.

One of the strengths of the study was the detailed individual-level information on water intake and potential confounding factors collected for this pregnancy cohort. Several of these confounders had considerable impact on the association between water intake and birth weight. For example, established risk factors for foetal growth measures such as maternal race, age, education, and household income attenuated the mean birth weight by $17-39 \%$ compared to the univariate total water intake model results. Although we did not find evidence that diabetes was a strong confounder in this analysis, results were slightly stronger when the birth weight data were restricted to non-diabetics (data not shown). We recognize that even after adjustment for confounding factors, there may be unmeasured aspects of maternal physiology or behaviour that affect both water intake and pregnancy outcome so that the water intake itself is not causally related to the outcomes. For example, previous research suggests that participants reporting no water intake also reported increased soft drink consumption and less fruit, vegetable and lowand medium-fat dairy product intake [17]. Although dietary information was not collected on this population, our detailed analysis of confounding likely led to indirect control of nutritional status to some degree through adjustment of confounders such as household income, education, and prenatal vitamin use. In addition, very 
Table 3 Small-for-gestational-age results ${ }^{a}$ for daily bottled, cold tap, total tap, and total water intake

\begin{tabular}{|c|c|c|c|c|}
\hline \multirow[b]{2}{*}{ Exposure } & \multicolumn{3}{|c|}{ Follow-up data } & \multirow{2}{*}{$\begin{array}{l}\begin{array}{l}\text { Average of baseline } \\
\text { and follow-up data }\end{array} \\
\text { Adjusted RR }(95 \% \mathrm{Cl})^{6}\end{array}$} \\
\hline & n (\%) & Unadjusted RR $(95 \% \mathrm{Cl})$ & Adjusted RR $(95 \% \mathrm{Cl})^{\mathrm{b}}$ & \\
\hline \multicolumn{5}{|l|}{ Bottled water } \\
\hline None & $448(26 \%)$ & 1 & 1 & 1 \\
\hline Any & $1258(74 \%)$ & $1.1(0.7,1.7)$ & $0.9(0.5,1.4)$ & $1.4(0.6,3.0)$ \\
\hline \multicolumn{5}{|c|}{ Cold tap water (ounces) ${ }^{c}$} \\
\hline $0-27$ & 403 (24\%) & 1 & 1 & 1 \\
\hline$>27-53$ & 439 (26\%) & $0.9(0.5,1.5)$ & $1.2(0.6,2.3)$ & $1.1(0.6,2.2)$ \\
\hline$>53-91$ & $429(25 \%)$ & $0.9(0.5,1.6)$ & $1.3(0.7,2.4)$ & $1.4(0.7,2.6)$ \\
\hline$>91$ & $439(26 \%)$ & $0.7(0.4,1.2)$ & $0.9(0.5,1.9)$ & $1.1(0.5,2.1)$ \\
\hline Per 20 ounce & & $1.0(0.9,1.0)$ & $1.0(0.9,1.1)$ & $1.0(0.9,1.1)$ \\
\hline \multicolumn{5}{|c|}{ Total tap water (ounces) ${ }^{c}$} \\
\hline $0-30$ & $423(25 \%)$ & 1 & 1 & 1 \\
\hline$>30-61$ & $426(25 \%)$ & $0.7(0.4,1.3)$ & $0.9(0.5,1.7)$ & $1.2(0.6,2.2)$ \\
\hline$>61-96$ & $476(28 \%)$ & $0.7(0.4,1.2)$ & $0.8(0.5,1.6)$ & $1.3(0.7,2.6)$ \\
\hline$>96$ & $380(22 \%)$ & $0.7(0.4,1.2)$ & $0.9(0.5,1.9)$ & $1.1(0.6,2.2)$ \\
\hline Per 20 ounce & & $1.0(0.9,1.1)$ & $1.0(0.9,1.1)$ & $1.0(0.9,1.1)$ \\
\hline \multicolumn{5}{|c|}{ Total water (ounces) $)^{c}$} \\
\hline $0-51$ & $434(25 \%)$ & 1 & 1 & 1 \\
\hline$>51-78$ & $418(25 \%)$ & $0.7(0.4,1.2)$ & $0.8(0.4,1.4)$ & $0.9(0.5,1.6)$ \\
\hline$>78-114$ & $424(25 \%)$ & $0.6(0.3,1.0)$ & $0.6(0.3,1.0)$ & $0.5(0.2,1.0)$ \\
\hline$>114$ & $427(25 \%)$ & $0.8(0.5,1.4)$ & $0.9(0.5,1.6)$ & $1.0(0.6,1.8)$ \\
\hline Per 20 ounce & & $1.0(0.9,1.1)$ & $1.0(0.9,1.1)$ & $1.0(0.9,1.1)$ \\
\hline
\end{tabular}

${ }^{a}$ Term SGA models restricted to infants born to non-Hispanic White, non-Hispanic Black, or Hispanic women

${ }^{\mathrm{b}}$ Adjusted for race, education level, annual household income, smoking, pre-pregnancy BMI, vitamin use, parity, employed during last 4 months, infant gender, and study site

${ }^{\mathrm{C}}$ Categorical exposure cutpoints for average data results were slightly higher than the follow-up data

$\mathrm{BMl}=$ body mass index; $\mathrm{RR}=$ risk ratio

few study participants $(0.3 \%)$ in our study population reported no water intake which should minimize the potential for confounding due to unhealthy lifestyles during pregnancy.

An additional study strength was the collection of multiple measures of water use during pregnancy which allowed for examination of water intake measures. This is potentially important for exposure assessment as previous studies have indicated that water use changes may occur during pregnancy [18]. It is not entirely clear, however, whether these changes are due to behavioural decisions related to perceived health benefits, physiologic changes such as increased thirst, or variation due to measurement error. Since 95\% of foetal growth occurs after the 20th gestational week, [19] we considered the follow-up data (collected during 20-24 gestational weeks) to be the most relevant data for examination of foetal growth measures and prematurity. However, we also examined water intake results based on a measure of the average follow-up/baseline data, but saw little difference in comparison to the follow-up data.

Although these data represent one of the most extensive water use data collective efforts to date in a reproductive epidemiological study, self-reported data are subject to recall error. Given the prospective nature of the data collection, differential error could not have occurred since the birth outcomes were unknown at the time the pregnant subjects reported the water consumption and information on confounding factors. Nonetheless, water intake is difficult to measure and may be subject to non-differential error. This may have reduced our statistical power and limited the ability to detect exposure-response relationships and effects small in magnitude (e.g., small changes in mean birth weight). Another limitation of the study was a narrow exposure gradient for bottled water intake which precluded examination of multiple exposure categories. In contrast, there was considerable variability in reported tap and total water intake across study subjects, but we did not have a truly unexposed (ie, those not consuming any water) reference group for the total tap and total water categories. The number of subjects $(0.3 \%)$ reporting no water intake in this population is less than that from other studies $(5-12 \%)$ [2,17,20].

Our study subjects were highly motivated, highly educated, and represented a low risk population, since they 
Table 4 Preterm delivery results for daily bottled, cold tap, total tap, and total water intake

\begin{tabular}{|c|c|c|c|c|}
\hline \multirow[b]{2}{*}{ Exposure } & \multicolumn{3}{|c|}{ Follow-up data } & \multirow{2}{*}{$\begin{array}{l}\begin{array}{l}\text { Average of baseline } \\
\text { and follow-up data }\end{array} \\
\text { Adjusted RR }(95 \% \mathrm{Cl})^{\mathrm{a}}\end{array}$} \\
\hline & n (\%) & Unadjusted RR (95\% Cl) & Adjusted RR $(95 \% \mathrm{Cl})^{a}$ & \\
\hline \multicolumn{5}{|l|}{ Bottled water } \\
\hline None & $483(25 \%)$ & 1 & 1 & 1 \\
\hline Any & $1458(75 \%)$ & $1.2(0.9,1.8)$ & $1.2(0.8,1.8)$ & $0.8(0.5,1.4)$ \\
\hline \multicolumn{5}{|c|}{ Cold tap water (ounces) ${ }^{b}$} \\
\hline $0-27$ & $472(24 \%)$ & 1 & 1 & 1 \\
\hline$>27-53$ & $494(25 \%)$ & $0.8(0.5,1.2)$ & $0.8(0.5,1.3)$ & $0.9(0.6,1.4)$ \\
\hline$>$ 53-91 & $485(25 \%)$ & $0.9(0.6,1.3)$ & $0.9(0.6,1.4)$ & $0.9(0.6,1.4)$ \\
\hline$>91$ & $490(25 \%)$ & $0.8(0.5,1.1)$ & $0.8(0.5,1.3)$ & $1.0(0.6,1.5)$ \\
\hline Per 20 ounce & & $1.0(0.9,1.0)$ & $0.9(0.7,1.3)$ & $0.9(0.6,1.3)$ \\
\hline \multicolumn{5}{|c|}{ Total tap water (ounces) } \\
\hline $0-30$ & $493(25 \%)$ & 1 & 1 & 1 \\
\hline$>30-61$ & $483(25 \%)$ & $0.8(0.5,1.2)$ & $0.8(0.5,1.2)$ & $1.0(0.6,1.5)$ \\
\hline$>61-96$ & $535(28 \%)$ & $0.7(0.5,1.1)$ & $0.8(0.5,1.2)$ & $1.2(0.8,1.9)$ \\
\hline$>96$ & $429(22 \%)$ & $0.9(0.6,1.3)$ & $1.0(0.6,1.5)$ & $0.9(0.6,1.4)$ \\
\hline Per 20 ounce & & $1.0(0.9,1.0)$ & $0.9(0.7,1.2)$ & $0.9(0.6,1.3)$ \\
\hline \multicolumn{5}{|c|}{ Total water (ounces) ${ }^{\mathrm{b}}$} \\
\hline $0-51$ & $491(25 \%)$ & 1.0 & 1.0 & 1.0 \\
\hline$>51-78$ & $478(25 \%)$ & $1.0(0.7,1.6)$ & $1.2(0.7,1.9)$ & $1.1(0.7,1.7)$ \\
\hline$>78-114$ & $481(25 \%)$ & $1.0(0.7,1.6)$ & $1.1(0.7,1.8)$ & $1.0(0.6,1.5)$ \\
\hline$>114$ & 478 (25\%) & $1.2(0.8,1.8)$ & $1.4(0.9,2.2)$ & $1.2(0.8,1.9)$ \\
\hline Per 20 ounce & & $1.0(1.0,1.1)$ & $1.1(0.9,1.4)$ & $1.1(0.8,1.5)$ \\
\hline
\end{tabular}

${ }^{a}$ Adjusted for race, education level, annual household income, smoking, pre-pregnancy BMl, vitamin use, parity, employed during last 4 months, infant gender, and study site

${ }^{\mathrm{b}}$ Categorical exposure cutpoints for average data results were slightly higher than the follow-up data

$\mathrm{BMI}=$ body mass index; $\mathrm{RR}=$ risk ratio

were actively seeking prenatal care during pregnancy and volunteered for this study. This may limit the generalizability of study findings and also raises the potential for bias among this highly motivated population if drinking water or other "healthful behaviours" are related to self-selection. The overall proportion of preterm births in the study population is lower than $(9 \%$ vs. 13\%) that reported in the United States in 2005 [21]. The prevalence of SGA (5\%) in our population based on birth weight deciles from United States population estimates was also lower than would be expected for the general population. This low risk population, therefore, limited our statistical power to detect associations due to the decreased frequency of adverse health outcomes (e.g., SGA) being considered. SGA may also include small births that are both pathologically growth restricted and some that are constitutionally small due to a variety of factors such as maternal ethnicity, parity, weight, height, etc. Therefore, the examination of SGA births is a potential limitation that could limit our ability to detect associations that may be present if some of these births represent constitutionally small births that are not truly growth restricted.

\section{Conclusions}

In conclusion, we found limited evidence of an association between specific measures of water intake and risk of adverse pregnancy outcomes such as SGA and PTD. This is in contrast to a previous study which reported an inverse association with water intake and risk of both SGA and PTD, [2] but is consistent with another study which found no association between water intake and risk of either PTD or low birth weight [3]. Despite limited statistical power, we did see some evidence of small increases in mean birth weight for higher levels of total water intake during pregnancy. This might warrant further examination in higher risk populations as this was the first study to examine this endpoint in relation to water intake.

\section{Acknowledgements}

We thank the obstetric practices and water utilities in the study areas for their help and cooperation, and we thank all of the women who participated in the Right from the Start study.

Funding: Supported jointly by the AWWA Research Foundation (AwwaRF: Project 2579) and the US Environmental Protection Agency (USEPA) under Cooperative Agreement nos. CR825625-01, CR827268-01, and CR828216-01, the Center for Environmental Health and Susceptibility (CEHS) at the 
University of North Carolina at Chapel Hill (P30E510126), US EPA STAR award RD-83184301-0, the Biostatistics for Research in Environmental Health training award of the National Institute of Environmental Health Sciences (5T32-ES07018), and the NHEERL-DESE cooperative training grant in Environmental Sciences Research (EPA CT8229471 and CR83323601).

\section{Author details}

'National Center for Environmental Assessment, US Environmental Protection Agency, Cincinnati, Ohio, USA. ${ }^{2}$ Division of Extramural Research and Training, National Institute of Environmental Health Sciences, Research Triangle Park, North Carolina, USA. ${ }^{3}$ Department of Community and Preventive Medicine, Mount Sinai School of Medicine, New York, New York, USA.

\section{Authors' contributions}

The design of cohort study was contributed by DAS. The scope and purpose of the paper was developed by all of the authors. The analyses were conducted by JMW and CSH. The text was written and reviewed by all of the authors.

\section{Competing interests}

The authors declare that they have no competing interests.

Received: 8 March 2010 Accepted: 24 August 2010

Published: 24 August 2010

\section{References}

1. Fowles ER: Prenatal nutrition and birth outcomes. J Obstet Gynecol Neonatal Nurs 2004, 33:809-822.

2. Savitz DA, Andrews KW, Pastore LM: Drinking water and pregnancy outcome in central North Carolina: source, amount, and trihalomethane levels. Environ Health Perspect 1995, 103:592-596.

3. Aggazzotti G, Righi E, Fantuzzi G, Biasotti B, Ravera G, Kanitz S, Barbone F, Sansebastiano G, Battaglia MA, Leoni V, et al: Chlorination by-products (CBPs) in drinking water and adverse pregnancy outcomes in Italy. $J$ Water Health 2004, 2:233-247.

4. Swan SH, Waller K, Hopkins B, Windham G, Fenster L, Schaefer C, Neutra RR: A prospective study of spontaneous abortion: relation to amount and source of drinking water consumed in early pregnancy. Epidemiology 1998, 9:126-133.

5. Shaw GM, Malcoe LH, Milea A, Swan SH: Chlorinated water exposures and congenital cardiac anomalies. Epidemiology 1990, 1:206-211.

6. Promislow JH, Makarushka CM, Gorman JR, Howards PP, Savitz DA, Hartmann KE: Recruitment for a community-based study of early pregnancy: the Right From The Start study. Paediatr Perinat Epidemiol 2004, 18:143-152.

7. Savitz DA, Singer PC, Herring AH, Hartmann KE, Weinberg HS, Makarushka C: Exposure to drinking water disinfection by-products and pregnancy loss. Am J Epidemiol 2006, 164:1043-1051.

8. Overpeck MD, Hediger ML, Zhang J, Trumble AC, Klebanoff MA: Birth weight for gestational age of Mexican American infants born in the United States. Obstet Gynecol 1999, 93:943-947.

9. Zhang J, Bowes WA Jr: Birth-weight-for-gestational-age patterns by race, sex, and parity in the United States population. Obstet Gynecol 1995, 86:200-208.

10. Committee on Nutritional Status During Pregnancy and Lactation, Institute of Medicine: Nutrition during Pregnancy: Part I: Weight Gain, Part II: Nutrients supplements. Washington, DC: Institute of Medicine 1990.

11. Fenster L, Hubbard AE, Swan SH, Windham GC, Waller K, Hiatt RA Benowitz N: Caffeinated beverages, decaffeinated coffee, and spontaneous abortion. Epidemiology 1997, 8:515-523.

12. Hoffman CS, Mendola P, Savitz DA, Herring AH, Loomis D, Hartmann KE, Singer PC, Weinberg HS, Olshan AF: Drinking water disinfection byproduct exposure and fetal growth. Epidemiology 2008, 19:729-737.

13. Hoffman CS, Mendola P, Savitz DA, Herring AH, Loomis D, Hartmann KE, Singer PC, Weinberg HS, Olshan AF: Drinking water disinfection byproduct exposure and duration of gestation. Epidemiology 2008 19:738-746.

14. Lewis C, Suffet IH, Hoggatt K, Ritz B: Estimated effects of disinfection byproducts on preterm birth in a population served by a single water utility. Environ Health Perspect 2007, 115:290-295.
15. Wright JM, Schwartz J, Dockery DW: Effect of trihalomethane exposure on fetal development. Occup Environ Med 2003, 60:173-180.

16. Wright JM, Schwartz J, Dockery DW: The effect of disinfection by-products and mutagenic activity on birth weight and gestational duration. Environ Health Perspect 2004, 112:920-925.

17. Popkin BM, Barclay DV, Nielsen SJ: Water and food consumption patterns of U.S. adults from 1999 to 2001. Obes Res 2005, 13:2146-2152.

18. Forssen UM, Wright JM, Herring AH, Savitz DA, Nieuwenhuijsen MJ, Murphy PA: Variability and predictors of changes in water use during pregnancy. J Expo Sci Environ Epidemiol 2009, 19:593-602.

19. Vorherr H: Factors influencing fetal growth. Am J Obstet Gynecol 1982, 142:577-588.

20. Shimokura GH, Savitz DA, Symanski E: Assessment of water use for estimating exposure to tap water contaminants. Environ Health Perspect 1998, 106:55-59.

21. Hamilton BE, Martin JA, Ventura SJ: Births: Preliminary data for 2008 National vital statistics reports; vol 58 no 16, Hyattsville, MD: National Center for Health Statistics. Released April 6, 2010

\section{Pre-publication history}

The pre-publication history for this paper can be accessed here: http://www.biomedcentral.com/1471-2393/10/48/prepub

doi:10.1186/1471-2393-10-48

Cite this article as: Wright et al:: The relationship between water intake and foetal growth and preterm delivery in a prospective cohort study. BMC Pregnancy and Childbirth 2010 10:48.

\section{Submit your next manuscript to BioMed Central and take full advantage of:}

- Convenient online submission

- Thorough peer review

- No space constraints or color figure charges

- Immediate publication on acceptance

- Inclusion in PubMed, CAS, Scopus and Google Scholar

- Research which is freely available for redistribution
C Biomed Central 